\title{
Type 2 immunity drives progression of NAFLD
}

A new study reveals contrasting effects of polarized immune responses in different tissues during obesity. According to research published in Science Translational Medicine, a type 2 immune response, characterized by accumulation of eosinophils, exacerbates progression of NAFLD and $\mathrm{NASH}$ yet is protective in metabolic dysfunction and obesity in mice. "Previously, it was thought that the inflammatory response driving adipogenesis was likely contributing to the development of NASH-associated fibrosis; however, this paper shows that while type 2 immunity plays a protective role in adipose tissues, it plays a pathogenic role in the liver by collaborating with transforming growth factor (TGF) $\beta$ to drive fibrosis," explains author Thomas Wynn.

NAFLD is now one of the most common progressive liver diseases, but the molecular and immunological mechanisms that underlie the progression of disease from simple steatosis to NAFLD, NASH, fibrosis and then cirrhosis remain unclear. Wynn and colleagues sought to examine the immunopathogenesis of NAFLD in more detail, investigating how polarized type 1 or type 2 immune responses affect NAFLD and fibrosis in mouse models.

The researchers first determined that $\mathrm{NASH}$ and fibrosis develop late in mice fed a high-fat diet (HFD), requiring long-term exposure to this diet. Mice fed a HFD for 15 weeks gained weight and developed hepatomegaly but did not show appreciable liver injury; however, after 40 weeks of a HFD, mice showed defining features of NASH, including hepatocyte ballooning and macrosteatosis. Next, they explored how a type 1 polarized immune response affects

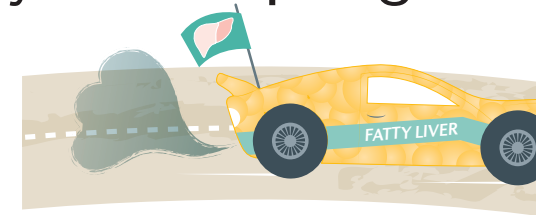

NAFLD development. Interestingly, $\left\|10^{-/-}\right\| 4^{-/-}$mice (as in, have a polarized type 1 immune response) fed a HFD were resistant to steatosis and NASH, but were susceptible to metabolic dysregulation in adipose tissue and obesity. This protection against NAFLD was associated with a hepatic IFN $\gamma$ signature. Accordingly, IFN $\gamma$-knockout mice fed a HFD had markedly increased hepatomegaly, steatosis, fibrosis and liver damage after 15 weeks on the diet - an accelerated progression of NAFLD.

Further characterization of NAFLD pathogenesis in the experimental mouse models revealed that $\mathrm{NASH}$-driven fibrosis was partly dependent on TGF $\beta$ : blockade of TGF $\beta$ (with a pan anti-TGF $\beta$ antibody) led to decreased liver fibrosis. In addition, the researchers found that TGF $\beta$ and IL-13 cooperatively drive fibrogenic pathways in the mouse NASH models. TGF $\beta$ blockade in I ng $^{-/-}$mice fed a HFD for 20 weeks had accelerated progression of advanced NAFLD, NASH and fibrosis compared with wild-type mice. Moreover, this NASH-driven fibrogenesis was characterized by severe hepatic eosinophilia localized in areas of marked lobular inflammation and macrosteatosis and was accompanied by increased $\mathrm{IL}-13$ production and upregulation of IL-13-responsive genes in the liver.

Crucially, experiments in an alternative mouse model of NASH (amylin liver NASH model diet, comparing wild-type and $/ \mathrm{fng}^{-/-}$mice) confirmed their findings: Ifng ${ }^{-/}$mice had increased hepatomegaly, fibrosis and liver damage compared with wild-type mice, alongside marked induction of type 2 inflammation and increased numbers of eosinophils in the liver. Moreover, analysis of liver biopsy samples from patients with NASH revealed that human NASH was characterized by type 2 eosinophilic inflammation.

"The specific role of eosinophils in NAFLD is unclear," notes Wynn. "Given their reported roles in metabolism, fibrosis, and liver healing or regeneration, further defining the functional role(s) of eosinophils and type 2 cytokines over the course of disease progression represents and exciting area of future research." Further research is needed to understand how the TGF $\beta$-IL-13 axis interacts with and regulates other inflammatory pathways that are active in the metabolic syndrome and NASH. "Given the pleiotropic roles for inflammation and cytokines in these processes and diseases, better characterizing the specific cellular targets and functional contributions of inflammatory pathways on these targets in the relevant organs will further shape our understanding and ability to intervene in both metabolic and fibrotic complications associated with obesity," concludes Wynn.

Katrina Ray

ORIGINAL ARTICLE Hart, K. M. et al. Type 2 immunity is protective in metabolic disese but exacerbates NAFLD collaboratively with TGF- $\beta$. Sci. Transl. Med. 9, eaal3694 (2017) 